\title{
Topological complexity of motion planning in projective product spaces
}

\author{
JESÚS GONZÁLEZ \\ MARK GRANT \\ ENRIQUE TORRES-GIESE \\ Miguel XicoténCATL
}

\begin{abstract}
We study Farber's topological complexity (TC) of Davis' projective product spaces (PPS's). We show that, in many nontrivial instances, the TC of PPS's coming from at least two sphere factors is (much) lower than the dimension of the manifold. This is in marked contrast with the known situation for (usual) real projective spaces for which, in fact, the Euclidean immersion dimension and TC are two facets of the same problem. Low TC-values have been observed for infinite families of nonsimply connected spaces only for $\mathrm{H}$-spaces, for finite complexes whose fundamental group has cohomological dimension at most 2 , and now in this work for infinite families of PPS's. We discuss general bounds for the TC (and the Lusternik-Schnirelmann category) of PPS's, and compute these invariants for specific families of such manifolds. Some of our methods involve the use of an equivariant version of TC. We also give a characterization of the Euclidean immersion dimension of PPS's through a generalized concept of axial maps or, alternatively (in an appendix), nonsingular maps. This gives an explicit explanation of the known relationship between the generalized vector field problem and the Euclidean immersion problem for PPS's.
\end{abstract}

55M30, 57R42; 68T40

\section{Introduction and notation}

As shown by Farber, Tabachnikov and Yuzvinsky in [10], the topological complexity (TC) and the Euclidean immersion dimension (Imm) of the $n$-dimensional real projective space $\mathrm{P}^{n}$ are related by

$$
\mathrm{TC}\left(\mathrm{P}^{n}\right)=\operatorname{Imm}\left(\mathrm{P}^{n}\right)-\epsilon(n)=2 n-\delta(n),
$$

where

$$
\epsilon(n)= \begin{cases}1 & n=1,3,7 \\ 0 & \text { otherwise }\end{cases}
$$


$\delta(n)=\mathrm{O}(\alpha(n))$ and $\alpha(n)$ denotes the number of ones in the binary expansion of $n$. It is natural to ask whether the nice phenomenon in the first equality in (1) is part of a general property of manifolds. Not only does this question have a negative answer, but even close relatives of real projective spaces fail to satisfy the first equality in (1). For instance, in view of the first author [14] and Astey, Davis and the first author [3], the failure holds for lens spaces whose fundamental group has torsion of the form $2^{e}$ for $e>1$. The same answer is observed in a forthcoming paper by two of the authors in which they study flag manifolds whose fundamental group is an elementary 2group of rank greater than 1 . This paper now shows that the list of counterexamples extends to Davis' projective product spaces, a family of manifolds giving a rather natural generalization of real projective spaces, and which, in particular, have $\mathbb{Z}_{2}$ as their fundamental groups (in the 'generic' case). Indeed, Theorem 3.8 in this paper shows that, in contrast to the second equality in (1), the topological complexity of a projective product space coming from at least two sphere factors can be much lower than the dimension of the manifold. Thus, in those cases, more than half the homotopy obstructions in the motion planning problem for $\mathrm{P}_{\bar{n}}$ are trivial (cf Remark 3.6 and the considerations after Theorem 3.8). To the authors' knowledge, this gives the first infinite family of nonsimply connected closed manifolds which are not $\mathrm{H}$-spaces and whose TC is lower than their dimension (cf García-Calcines and Vandembroucq [11] and Lupton and Scherer [24]; the upper bound in Costa and Farber [6, Theorem 3] should be noted, too).

In the rest of this introductory section we set up notation and recall needed preliminary results. We use the reduced form of the Schwarz genus (also called sectional category, and denoted by secat) of a fibration, ie a trivial fibration has zero genus. In particular, we consider the reduced form of the Lusternik-Schnirelmann category (cat) and that of Farber's topological complexity (TC) of a space $X$-the latter being the reduced Schwarz genus of the double evaluation map $X^{[0,1]} \rightarrow X \times X$ sending a path $\gamma:[0,1] \rightarrow X$ to the pair $(\gamma(0), \gamma(1))$. Thus, $\operatorname{cat}(X)=\mathrm{TC}(X)=0$ for a contractible space $X$. We will also assume the reader is familiar with Davis [7], and we next briefly recall the required results from that paper.

We let $\bar{n}$ stand for an $r$-tuple $\left(n_{1}, \ldots, n_{r}\right)$ of positive integers with $n_{1} \leq \cdots \leq n_{r}$. We consider the diagonal action of $\mathbb{Z}_{2}$ on $S_{\bar{n}}:=S^{n_{1}} \times \cdots \times S^{n_{r}}$, and let $\mathrm{P}_{\bar{n}}$ denote the resulting orbit space (so $\mathrm{P}_{\left(n_{1}\right)}$ is the usual real projective space $\mathrm{P}^{n_{1}}$ ). We set $|\bar{n}|:=\operatorname{dim}\left(\mathrm{P}_{\bar{n}}\right)=\operatorname{dim}\left(S_{\bar{n}}\right)=\sum n_{i}$ and $\ell(\bar{n})=r$. The real line bundle associated to the obvious covering $S_{\bar{n}} \rightarrow \mathrm{P}_{\bar{n}}$, denoted by $\xi_{\bar{n}}$ and called the canonical line bundle over $\mathrm{P}_{\bar{n}}$, can be used to identify the stable class of the tangent bundle $\tau_{\mathrm{P}_{\bar{n}}}$ since

$$
\tau_{\mathrm{P}_{\bar{n}}} \oplus r \varepsilon \approx(|\bar{n}|+r) \xi_{\bar{n}}
$$


Here $\varepsilon$ stands for a trivial line bundle.

The total space of the $k$-fold iterated Whitney sum of $\xi_{\bar{n}}$ is given by the Borel construction $k \xi_{\bar{n}}=S_{\bar{n}} \times_{\mathbb{Z}_{2}} \mathbb{R}^{k}$. In particular, the projectivization of $k \xi_{\bar{n}}$ is given by

$$
P\left(k \xi_{\bar{n}}\right)=\mathrm{P}_{\bar{n}} \times \mathrm{P}^{k-1} .
$$

The diagonal inclusion $S^{n_{1}} \hookrightarrow S_{\bar{n}}$ and the projection onto the first factor $S_{\bar{n}} \rightarrow S^{n_{1}}$ induce corresponding maps $j: \mathrm{P}^{n_{1}} \hookrightarrow \mathrm{P}_{\bar{n}}$ and $p: \mathrm{P}_{\bar{n}} \rightarrow \mathrm{P}^{n_{1}}$ satisfying

$$
j^{*}\left(\xi_{\bar{n}}\right) \approx \xi_{n_{1}}, \quad p^{*}\left(\xi_{n_{1}}\right) \approx \xi_{\bar{n}}, \quad p \circ j=\mathrm{Id} .
$$

For $2 \leq i \leq r$ there are mod 2 cohomology classes $x_{i}$ in $\mathrm{P}_{\bar{n}}$ with $\operatorname{dim}\left(x_{i}\right)=n_{i}$ such that the mod 2 cohomology ring of $\mathrm{P}_{\bar{n}}$ is given by

$$
H^{*}\left(\mathrm{P}_{\bar{n}} ; \mathbb{Z}_{2}\right)=H^{*}\left(\mathrm{P}^{n_{1}} ; \mathbb{Z}_{2}\right) \otimes \Lambda\left[x_{2}, \ldots, x_{r}\right],
$$

where $\Lambda$ denotes an exterior algebra with the only exception that, if $n_{1}$ is even, then $x_{i}^{2}=x^{n_{1}} x_{i}$ whenever $n_{i}=n_{1}$. Here $x \in H^{1}\left(\mathrm{P}^{n_{1}} ; \mathbb{Z}_{2}\right)$ satisfies

$$
x=w_{1}\left(\xi_{\bar{n}}\right) \text {, but all classes } x_{i} \text { restrict trivially under the inclusion } j .
$$

We also need the concept of 'generalized axial map' as defined in [3]: For a real vector bundle $\alpha$ over a space $X$, we let $S(\alpha)$ and $P(\alpha)$ stand, respectively, for the sphere and projectivized bundles associated to $\alpha$. Let $h_{\alpha}$ denote the Hopf line bundle over $P(\alpha)$ splitting off $\pi^{*}(\alpha)$, where $\pi: P(\alpha) \rightarrow X$ is the projection. A Hopf-type map ${ }^{1}$ for $\alpha$ is any continuous map $P(\alpha) \rightarrow \mathrm{P}^{N}$ for which the composite $P(\alpha) \rightarrow \mathrm{P}^{N} \hookrightarrow \mathrm{P}^{\infty}$ classifies $h_{\alpha}$. In particular, (3) allows us to talk about Hopf-type maps defined on products of the form $\mathrm{P}_{\bar{n}} \times \mathrm{P}^{s}$.

Acknowledgements The authors wish to thank Peter Landweber for useful suggestions on a preliminary version of this paper. The first author was supported by CONACYT Grant 102783 during the time this research was conducted. The fourth author would like to thank CONACYT grant 168349 and ABACUS, CONACYT grant EDOMEX 2011-C01-165873.

\section{Immersion dimension and axial maps}

Consider a pair of sequences $\bar{n}=\left(n_{1}, \ldots, n_{r}\right)$ and $\bar{m}=\left(m_{1}, \ldots, m_{s}\right)$.

\footnotetext{
${ }^{1}$ This is called an 'axial map' in [3], but we have to modify the name in view of Definition 2.1 in the next section.
} 
Definition 2.1 A continuous map $\alpha: \mathrm{P}_{\bar{n}} \times \mathrm{P}_{\bar{m}} \rightarrow \mathrm{P}^{\infty}$ is said to be axial if its restriction to each of the axes classifies the corresponding canonical bundle. By (6) this means that $\alpha$ corresponds to the class $x \otimes 1+1 \otimes x$. A continuous map $\mathrm{P}_{\bar{n}} \times \mathrm{P}_{\bar{m}} \rightarrow \mathrm{P}^{L}$ is called axial if the composite $\mathrm{P}_{\bar{n}} \times \mathrm{P}_{\bar{m}} \rightarrow \mathrm{P}^{L} \hookrightarrow \mathrm{P}^{\infty}$ is axial.

Remark 2.2 By (4), the existence of an axial map $\mathrm{P}_{\bar{n}} \times \mathrm{P}_{\bar{m}} \rightarrow \mathrm{P}^{L}$ depends only on $n_{1}$ and $m_{1}$. In particular, according to [10], if $n_{1}=m_{1}, \mathrm{TC}\left(\mathrm{P}^{n_{1}}\right)$ is the minimal integer $L$ for which there is an axial map $\mathrm{P}_{\bar{n}} \times \mathrm{P}_{\bar{m}} \rightarrow \mathrm{P}^{L}$. In any case, an axial map $\mathrm{P}_{\bar{n}} \times \mathrm{P}_{\bar{m}} \rightarrow \mathrm{P}^{L}$ can exist only if $L \geq \max \left\{n_{1}, m_{1}\right\}$.

A slightly weaker concept of axiality arises by requiring that the restriction of the map $\alpha: \mathrm{P}_{\bar{n}} \times \mathrm{P}_{\bar{m}} \rightarrow \mathrm{P}^{\infty}$ to $j \times j: \mathrm{P}^{n_{1}} \times \mathrm{P}^{m_{1}} \hookrightarrow \mathrm{P}_{\bar{n}} \times \mathrm{P}_{\bar{m}}$ is axial in the usual sense. Yet, nothing is lost with respect to the more restrictive Definition 2.1 if we only care (as we will in this subsection) about the existence of such maps. Indeed, in view of (5), the only potential problem arises when $n_{2}=1$ or $m_{2}=1$. To fix ideas, assume $n_{2}=\cdots=n_{\ell}=1<n_{\ell+1}(\ell \leq r)$. Then, the restriction of $\alpha$ to its first axis might conceivably correspond to a class of the form $x+\sum_{i=2}^{\ell} \epsilon_{i} x_{i}$. Although such a situation is perfectly attainable, it can be easily fixed. Indeed, [7, Theorem 2.20] asserts that, under the present conditions, $\mathrm{P}_{\bar{n}}$ is homeomorphic to $\left(S^{1}\right)^{\ell-1} \times \mathrm{P}_{\bar{q}}$, where $\bar{q}=\left(1, n_{\ell+1}, \ldots, n_{r}\right)$. Thus, unless $m_{2}=1$ (in which case the following adjustment would have to be made on the second axis, too), the required axial map is given by the composite $\mathrm{P}_{\bar{n}} \times \mathrm{P}_{\bar{m}} \rightarrow \mathrm{P}_{\bar{n}} \times \mathrm{P}_{\bar{m}} \rightarrow \mathrm{P}^{\infty}$ where the first map is $\gamma \times 1$, the second map is $\alpha$ and $\gamma$ is the projection $\mathrm{P}_{\bar{n}} \rightarrow \mathrm{P}_{\bar{q}}$ followed by the inclusion $\mathrm{P}_{\bar{q}} \hookrightarrow \mathrm{P}_{\bar{n}}$.

As a consequence of Remark 2.2, the nice relationship between TC and the existence of suitable axial maps between (usual) real projective spaces cannot hold for a $\mathrm{P}_{\bar{n}}$ with $\ell(\bar{n})>1$. Yet, the axial map approach ${ }^{2}$ can be used to characterize the immersion dimension of $\mathrm{P}_{\bar{n}}$ in a suitable range of dimensions. Indeed, the following are standard consequences of [7] and Sanderson [28]:

(I) The existence of a smooth immersion $\mathrm{P}_{\bar{n}} \uparrow \rightarrow \mathbb{R}^{M}$ implies the existence of an axial map $\mathrm{P}^{n_{1}} \times \mathrm{P}^{|\bar{n}|+r-1} \rightarrow \mathrm{P}^{M+r-1}$.

(II) The converse of (I) holds provided that $\mathrm{P}_{\bar{n}}$ is not stably parallelizable and $n_{1}<2(M-|\bar{n}|)$.

We will now elaborate on the previous facts from a purely 'projective-product' viewpoint, without relying on the connection through the generalized vector field problem.

${ }^{2}$ The next two sections deal with $\operatorname{TC}\left(\mathrm{P}_{\bar{n}}\right)$. 
Proposition 2.3 The existence of an immersion $\mathrm{P}_{\bar{n}} \rightarrow \mathbb{R}^{M}$ implies the existence of a Hopf-type map $\mathrm{P}_{\bar{n}} \times \mathrm{P}^{|\bar{n}|+r-1} \rightarrow \mathrm{P}^{M+r-1}$.

Proof Let $\varepsilon$ be the trivial line bundle over $\mathrm{P}_{\bar{n}}$ and $v$ the normal bundle of the given immersion. From (2) we have the composite

$$
(|\bar{n}|+r) \xi_{\bar{n}} \hookrightarrow(|\bar{n}|+r) \xi_{\bar{n}} \oplus v=\left(\tau_{\mathrm{P}_{\bar{n}}} \oplus r \varepsilon\right) \oplus v=(M+r) \varepsilon .
$$

The required Hopf-type map is given by the composite

$$
\mathrm{P}_{\bar{n}} \times \mathrm{P}^{|\bar{n}|+r-1}=P((|\bar{n}|+r) \xi \bar{n}) \hookrightarrow P((M+r) \varepsilon)=\mathrm{P}_{\bar{n}} \times \mathrm{P}^{M+r-1} \stackrel{\text { proj }}{\longrightarrow} \mathrm{P}^{M+r-1}
$$

(cf [3, Section 2]).

Remark 2.4 The converse of Proposition 2.3 can be proved (under an additional hypothesis) in terms of a standard application of Haefliger-Hirsch homotopy approximation of monomorphisms by skew-maps in the metastable range (see Haefliger and Hirsch [18], compare with Adem, Gitler and James [2] or [3, Corollary 2.8]). Indeed, the axial map in the conclusion of Proposition 2.3 is double covered by a $\mathbb{Z}_{2}$-equivariant map

$$
S\left((|\bar{n}|+r) \xi_{\bar{n}}\right)=S_{\bar{n}} \times_{\mathbb{Z}_{2}} S^{|\bar{n}|+r-1} \rightarrow S^{M+r-1} .
$$

This and the projection $(|\bar{n}|+r) \xi_{\bar{n}} \rightarrow \mathrm{P}_{\bar{n}}$ determine a map $S\left((|\bar{n}|+r) \xi_{\bar{n}}\right) \rightarrow \mathrm{P}_{\bar{n}} \times S^{M+r-1}$ which, after radial extension, yields a skew map $(|\bar{n}|+r) \xi_{\bar{n}} \rightarrow(M+r) \varepsilon$ over $\mathrm{P}_{\bar{n}}$. Theorem 1.2 in [18] claims that the latter map can be skew-deformed to a bundle monomorphism $\phi:(|\bar{n}|+r) \xi_{\bar{n}} \hookrightarrow(M+r) \varepsilon$ provided

$$
3|\bar{n}|<2 M \text {. }
$$

Coker $(\phi)$ is then an $(M-|\bar{n}|)$-dimensional bundle which, after taking into account (2) and cancelling $r$ trivial sections, yields an isomorphism $\tau_{\mathrm{P}_{\bar{n}}} \oplus \operatorname{Coker}(\phi)=M \varepsilon$. Thus Hirsch [19] asserts that $\operatorname{Coker}(\phi)$ is the normal bundle of an immersion, as required.

Of course, the hypothesis (7) is much stronger than the arithmetical condition in (II), a hypothesis where $n_{1}$ plays a more relevant role (and which is in accordance with Remark 2.2).

Proposition 2.5 There is a Hopf-type map $\mathrm{P}_{\bar{n}} \times \mathrm{P}^{|\bar{n}|+r-1} \rightarrow \mathrm{P}^{M+r-1}$ if and only if there is an axial map $\mathrm{P}^{n_{1}} \times \mathrm{P}^{|\bar{n}|+r-1} \rightarrow \mathrm{P}^{M+r-1}$. 
Proof In view of (6), it suffices to check that the map $\mathrm{P}_{\bar{n}} \times \mathrm{P}^{|\bar{n}|+r-1} \rightarrow \mathrm{P}^{\infty}$ that classifies the Hopf line bundle $h_{(|\bar{n}|+r) \xi_{\bar{n}}}$ corresponds to $x \otimes 1+1 \otimes x$. For this purpose, we may assume without loss of generality that the given Hopf-type map arises from an immersion as in Proposition 2.3 (say for a large enough $M$-this is irrelevant for the intended goal). Then, with the notation of that result, we see from (4) that, by restricting the isomorphism $(|\bar{n}|+r) \xi_{\bar{n}} \oplus v=(M+r) \varepsilon$ under the inclusion $j: \mathrm{P}^{n_{1}} \hookrightarrow \mathrm{P}_{\bar{n}}$, we get a Hopf-type map

$$
\mathrm{P}^{n_{1}} \times \mathrm{P}^{|\bar{n}|+r-1}=P\left((|\bar{n}|+r) \xi_{n_{1}}\right) \hookrightarrow P\left((|\bar{n}|+r) \xi_{\bar{n}}\right)=\mathrm{P}_{\bar{n}} \times \mathrm{P}^{|\bar{n}|+r-1} \rightarrow \mathrm{P}^{M+r-1},
$$

which, as proved in [2], must also be an axial map. Thus, (6) implies that $h_{(|\bar{n}|+r) \xi_{\bar{n}}}$ corresponds, under the identification $P\left((|\bar{n}|+r) \xi_{\bar{n}}\right)=\mathrm{P}_{\bar{n}} \times \mathrm{P}^{|\bar{n}|+r-1}$ in (3), to a class of the form

$$
1 \otimes x+\left(x+\sum \mu_{i} x_{i}\right) \otimes 1
$$

where the summation runs over indices $i$ with $n_{i}=1$, and each $\mu_{i}$ is either 0 or 1 . But the first isomorphism in (4) and the naturality of the construction of Hopf line bundles imply $\mu_{i}=0$ for all relevant $i$.

Example 2.6 The arithmetical hypothesis in (II) above is superfluous when $\ell(\bar{n})=1$, but it is needed if $\ell(\bar{n})>1$. From our perspective, such a phenomenon is due to the fact that, although an immersion $\mathrm{P}^{n} \uparrow \rightarrow \mathbb{R}^{M}$ can exist only within Haefliger's metastable range (ie with $3 n<2 M$, see [2]), as noted in [7], a projective product space $\mathrm{P}_{\bar{n}}$ with $\ell(\bar{n})>1$ usually admits (very) low-codimension Euclidean immersions-compare to Remark 2.8 below. For instance ${ }^{3}$, the nonparallelizable $\mathrm{P}_{(12,14)}$ does not immerse in $\mathbb{R}^{30}$ in view of [7, Theorem 3.4], [28, Lemma 2.2], and Lam and Randall [23] (in that order), but the existence of the corresponding axial map in (I) is obtained in [23] through a Postnikov tower argument.

Despite Example 2.6, the method of proof of the main result in [2] yields the following.

Proposition 2.7 If $\operatorname{gd}\left(-(|\bar{n}|+r) \xi_{n_{1}}\right)>\left\lceil\left(n_{1}+1\right) / 2\right\rceil$, then the arithmetical hypothesis in (II) is superfluous.

Proof Seeking a contradiction, assume that, for some $M$, there is an axial map $\mathrm{P}^{n_{1}} \times \mathrm{P}^{|\bar{n}|+r-1} \rightarrow \mathrm{P}^{M+r-1}$ but that the nonstably parallelizable $\mathrm{P}_{\bar{n}}$ does not immerse in $\mathbb{R}^{M}$. Without loss of generality we can assume that $M=\operatorname{imm}\left(\mathrm{P}_{\bar{n}}\right)-1>|\bar{n}|$. Then, [7, Theorem 3.4] gives

$$
M-|\bar{n}|=\operatorname{imm}\left(\mathrm{P}_{\bar{n}}\right)-|\bar{n}|-1=\operatorname{gd}\left(-(|\bar{n}|+r) \xi_{n_{1}}\right)-1 \geq\left\lceil\frac{n_{1}+1}{2}\right\rceil,
$$

${ }^{3}$ We thank Kee Lam for kindly pointing out this example. 
which amounts to having the arithmetical hypothesis in (II).

Remark 2.8 By the same line of reasoning as in Example 2.6, it follows from Adams [1] that, for any large $n_{1}$, there are instances of spaces $\mathrm{P}_{\left(n_{1}, \ldots, n_{r}\right)}$ for which the hypothesis in Proposition 2.7 fails.

It is worth mentioning that, for $n_{1} \leq 9$, the arithmetical hypothesis in (II) above is superfluous ${ }^{4}$. As in the proof of Proposition 2.7, such an assertion can be verified by checking that, in the indicated range, there is no axial map $\mathrm{P}^{n_{1}} \times \mathrm{P}^{|\bar{n}|+r-1} \rightarrow \mathrm{P}^{M+r-1}$ with $M=\operatorname{imm}\left(\mathrm{P}_{\bar{n}}\right)-1>|\bar{n}|$. Indeed, under the current hypothesis, such an axial map is prevented by the relation

$$
(x+y)^{M+r} \neq 0,
$$

where $x$ and $y$ denote respectively the generators of the mod 2 cohomology groups $H^{1}\left(\mathrm{P}^{n_{1}} ; \mathbb{Z}_{2}\right)$ and $H^{1}\left(\mathrm{P}^{|\bar{n}|+r-1} ; \mathbb{Z}_{2}\right)$. Explicitly, the basis element $x^{g} y^{|\bar{n}|+r-1} \in$ $H^{*}\left(\mathrm{P}^{n_{1}} \times \mathrm{P}^{|\bar{n}|+r-1} ; \mathbb{Z}_{2}\right)$ appears in the expansion of (8) with coefficient

$$
\left(\begin{array}{c}
|\bar{n}|+r+g-1 \\
g
\end{array}\right)
$$

where $g=\operatorname{gd}\left(-(|\bar{n}|+r) \xi_{n_{1}}\right)$. But, under the current hypothesis, a direct verification using Lam [22] (or, alternatively, [7, Table 4.4 or Proposition 4.5]) shows that (9) is odd. For instance, consider the case $n_{1}=6$, where the assumption that $\mathrm{P}_{\bar{n}}$ is not stably parallelizable means $|\bar{n}|+r \not \equiv 0 \bmod 8$. Then, [7, Table 4.4] gives $g=(6,6,5,4,3,2,1)$ for $|\bar{n}|+r \equiv(1,2,3,4,5,6,7) \bmod 8$. So

$$
\left(\begin{array}{c}
|\bar{n}|+r+g-1 \\
g
\end{array}\right) \equiv\left(\left(\begin{array}{l}
6 \\
6
\end{array}\right),\left(\begin{array}{l}
7 \\
6
\end{array}\right),\left(\begin{array}{l}
7 \\
5
\end{array}\right),\left(\begin{array}{l}
7 \\
4
\end{array}\right),\left(\begin{array}{l}
7 \\
3
\end{array}\right),\left(\begin{array}{l}
7 \\
2
\end{array}\right),\left(\begin{array}{l}
7 \\
1
\end{array}\right)\right) \equiv 1 \bmod 2 .
$$

We close this subsection by remarking that, just as the situation in Example 2.6 for the condition $n_{1}<2(M-|\bar{n}|)$, the hypothesis that $\mathrm{P}_{\bar{n}}$ is not stably parallelizable is also needed in (II). Yet, the full TC-axial picture is well understood in the stably parallelizable case. In fact, the situation is entirely similar to that in the classical case with $\ell(\bar{n})=1$, where there are well-known axial maps $\mathrm{P}^{n} \times \mathrm{P}^{n} \rightarrow \mathrm{P}^{n}$ for $n=1,3,7$, but of course no immersion $\mathrm{P}^{n} \rightarrow \mathbb{R}^{n}$. Namely, since the immersion dimension of a stably parallelizable $\mathrm{P}_{\bar{n}}$ is $|\bar{n}|+1$, there is an axial map $\mathrm{P}_{\bar{n}} \times \mathrm{P}^{|\bar{n}|+r-1} \rightarrow \mathrm{P}^{|\bar{n}|+r}$. But there is a finer (and optimal) axial map

$$
\mathrm{P}_{\bar{n}} \times \mathrm{P}^{|\bar{n}|+r-1} \rightarrow \mathrm{P}^{|\bar{n}|+r-1}
$$

\footnotetext{
${ }^{4}$ Kee Lam has brought to the authors' attention that the smallest case where the arithmetical hypothesis in (II) is actually needed takes place when $n_{1}=10$.
} 
(which cannot come from an immersion). Indeed, as shown in [7], the stable parallelizability of $\mathrm{P}_{\bar{n}}$ means that the exponent in the highest 2-power dividing $|\bar{n}|+r$ is no less than $\phi\left(n_{1}\right)$ - the number of positive integers less than or equal to $n_{1}$ and which are congruent to $0,1,2$, or 4 mod 8 . Therefore, classical work of Hurwitz, Radon, and Eckmann on the so-called Hurwitz-Radon matrix equations gives in fact a nonsingular bilinear map $\mathbb{R}^{n_{1}+1} \times \mathbb{R}^{|\bar{n}|+r} \rightarrow \mathbb{R}^{|\bar{n}|+r}$ and, in view of Remark 2.2, an axial map of the form (10). An intriguing possibility is that explicit 'linear' formulæ leading to an axial map (10) could be deduced from a refinement of the Clifford-algebra input in the Hurwitz-Radon number-without relying on Remark 2.2.

\section{Topological complexity}

In this section we give several general estimates for $\mathrm{TC}\left(\mathrm{P}_{\bar{n}}\right)$. We find $\operatorname{TC}\left(\mathrm{P}_{\bar{n}}\right)<\operatorname{dim}\left(\mathrm{P}_{\bar{n}}\right)$ in certain cases, indicating that a simple relation to immersion dimension such as (1) does not hold for these manifolds. We also compute the exact value of $\operatorname{TC}\left(\mathrm{P}_{\bar{n}}\right)$ in many cases (Proposition 3.7), and give evidence toward the appealing possibility that $\operatorname{TC}\left(\mathrm{P}_{\bar{n}}\right)$ would depend mostly on $\operatorname{TC}\left(\mathrm{P}^{n_{1}}\right)$ and $\ell(\bar{n})$.

Let $\bar{\infty}$ stand for the $r$-tuple $(\infty, \ldots, \infty)$, and let $\mathrm{P}_{\bar{\infty}}$ denote the quotient of $\prod_{r} S^{\infty}$ by the diagonal action of $\mathbb{Z}_{2}$ (with the antipodal action on each factor). Note that $\mathrm{P}_{\bar{\infty}}$ is an Eilenberg-Mac Lane space $K\left(\mathbb{Z}_{2}, 1\right)$ containing $\mathrm{P}_{\bar{n}}$.

Lemma 3.1 There is a $C W$ decomposition for $\mathrm{P}_{\bar{\infty}}$ whose $n_{1}$-skeleton is contained in $\mathrm{P}_{\bar{n}}$.

Proof Let $e_{+}^{0} \cup e_{-}^{0} \cup \cdots \cup e_{+}^{m} \cup e_{-}^{m}$ be the usual $\mathbb{Z}_{2}$-equivariant cell structure on a sphere $S^{m}$, and consider the resulting product structure

$$
S_{\bar{n}}=\bigcup e_{ \pm}^{i_{1}} \times \cdots \times e_{ \pm}^{i_{r}}
$$

If $\tau$ stands for the generator of $\mathbb{Z}_{2}$, then a cell structure on $\mathrm{P}_{\bar{n}}$ can be formed by identifying a cell $e_{ \pm}^{i_{1}} \times \cdots \times e_{ \pm}^{i_{r}}$ in (11) with the corresponding cell $\tau \cdot\left(e_{ \pm}^{i_{1}} \times \cdots \times e_{ \pm}^{i_{r}}\right)$. If $\ell(\bar{m})=\ell(\bar{n})$ and $n_{i} \leq m_{i}$, then the inclusion $\mathrm{P}_{\bar{n}} \hookrightarrow \mathrm{P}_{\bar{m}}$ contains the $n_{1}$-skeleton of $\mathrm{P}_{\bar{m}}$. Thus the required cell structure in $\mathrm{P}_{\bar{\infty}}$ is the inductive one under the above inclusions.

We are indebted to Sergey Melikhov for pointing out (in Melikhov [25]) the proof of the following fact. 
Proposition 3.2 Let $M^{m}$ and $N^{n}$ be closed smooth manifolds, and let $C^{\infty}(M, N)$ denote the space of smooth maps in the Whitney $C^{\infty}$-topology. Then for $f: M \rightarrow N$ in a dense subset of $C^{\infty}(M, N)$, the fibers $f^{-1}(y)$ with $y \in N$ are all polyhedra of dimension less than or equal to $\max (m-n, 0)$.

Proof First we note that the set of triangulable maps is dense in $C^{\infty}(M, N)$. Recall that a smooth map $f: M \rightarrow N$ is triangulable if there exists a PL map $g: K \rightarrow L$ between PL manifolds, and homeomorphisms $h: M \rightarrow K$ and $h^{\prime}: N \rightarrow L$ such that $g \circ h=h^{\prime} \circ f$. By Verona's proof of Thom's triangulation conjecture [29], we know that all proper, topologically stable maps $f: M \rightarrow N$ are triangulable. By the ThomMather theorem (a full proof of which appears in Gibson, Wirthmüller, du Plessis and Looijenga [12]), such maps form an open dense subset of $C^{\infty}(M, N)$.

Next, we note that the fibers $f^{-1}(y)$ of a triangulable map $f: M \rightarrow N$ are all polyhedra (they are homeomorphic to simplicial complexes). For given $y \in N$, we may choose a triangulation $h^{\prime}: N \rightarrow L$ as above with $h^{\prime}(y)$ a vertex of $L$. Then $f^{-1}(y)$ is homeomorphic with $g^{-1}\left(h^{\prime}(y)\right)$, a subcomplex of $K$.

Finally, we claim that for $f: M \rightarrow N$ in an open dense subset of the space $C^{\infty}(M, N)$, the fibers $f^{-1}(y)$ all have covering dimension less than or equal to $\max (m-n, 0)$. Intersecting this set with the set of proper, topologically stable maps, we find an open dense set of maps whose fibers are all polyhedra of covering dimension less than or equal to $\max (m-n, 0)$. Since covering dimension is a topological property, this proves the Proposition.

The proof of the final claim follows from the multijet transversality theorem by Golubitsky and Guillemin [13], which implies that for an open dense set of mappings $f: M \rightarrow N$, the fibers $f^{-1}(y)$ all have the structure of a smooth submanifold of $M$ of dimension $\max (m-n, 0)$ away from at most finitely many isolated singular points.

Theorem 3.3 $\operatorname{TC}\left(\mathrm{P}_{\bar{n}}\right) \leq 2|\bar{n}|-n_{1}+1$ for $\ell(\bar{n})>1$. On the other hand, the following numbers are equal, giving a lower bound for $\mathrm{TC}\left(\mathrm{P}_{\bar{n}}\right)$ :

- the Schwarz genus of the obvious double cover $S_{\bar{n}} \times_{\mathbb{Z}_{2}} S_{\bar{n}} \rightarrow \mathrm{P}_{\bar{n}} \times \mathrm{P}_{\bar{n}}$;

- the smallest integer $L$ for which $(L+1) \xi_{\bar{n}} \otimes \xi_{\bar{n}}$ admits a nowhere zero section;

- the smallest integer $L$ for which there is an axial map $\mathrm{P}_{\bar{n}} \times \mathrm{P}_{\bar{n}} \rightarrow \mathrm{P}^{L}$;

- $\mathrm{TC}\left(\mathrm{P}^{n_{1}}\right)$.

Proof It follows from Remark 2.2 and the first two conditions in (4) that the number described in each of the first three items does not change if $\bar{n}$ is replaced by $n_{1}$ (for 
the first item we use the fact that the indicated double cover is the sphere bundle associated to $\left.\xi_{\bar{n}} \otimes \xi_{\bar{n}}\right)$. Therefore, the equality of the four listed numbers follows from [10, Theorem 6.1]. The fact that they give a lower bound for $\operatorname{TC}\left(\mathrm{P}_{\bar{n}}\right)$ follows from the third condition in (4) and the behavior of TC under retracts.

We use the argument by the second author in [16, Corollary 4.5] (which is inspired in turn by Oprea and Walsh [27]) to prove the upper bound in this theorem. Set $L=2|\bar{n}|-n_{1}+1$. By (1) and Remark 2.2, we can choose an axial map $q: \mathrm{P}_{\bar{n}} \times \mathrm{P}_{\bar{n}} \rightarrow \mathrm{P}^{L}$. Since the axial condition is homotopical, we can assume first that $q$ is smooth and then, by Proposition 3.2, that for each $z \in \mathrm{P}^{L}$ the inverse image $q^{-1}(z)$ is homeomorphic to a CW complex of dimension at most $n_{1}-1$. Then, the axiality of $q$ implies that the image of the class $x$ in (6) under the composite

$$
q^{-1}(z) \hookrightarrow \mathrm{P}_{\bar{n}} \times \mathrm{P}_{\bar{n}} \stackrel{\pi_{i}}{\longrightarrow} \mathrm{P}_{\bar{n}}
$$

is independent of the projection $\pi_{i}: \mathrm{P}_{\bar{n}} \times \mathrm{P}_{\bar{n}} \rightarrow \mathrm{P}_{\bar{n}}(i=1,2)$ used. In fact, Lemma 3.1 and the dimensionality assumption on $q^{-1}(z)$ imply that the actual homotopy type of (12) is independent of $i$. The result then follows from [16, Lemma 2.5 and Theorem 4.3].

Of course, part of the argument for the lower bound in Theorem 3.3 actually yields $\mathrm{TC}\left(\mathrm{P}^{n_{1}}\right) \leq \mathrm{TC}\left(\mathrm{P}_{\left(n_{1}, n_{2}\right)}\right) \leq \cdots \leq \mathrm{TC}\left(\mathrm{P}_{\left(n_{1}, \ldots, n_{r-1}\right)}\right) \leq \mathrm{TC}\left(\mathrm{P}_{\bar{n}}\right)$. On the other hand, the argument proving the upper bound uses and corrects the proof of [16, Corollary 4.5] which, instead of using Proposition 3.2, is based on an assertion about approximating axial maps by submersions. But such a claim is false in general, as illustrated next.

Example 3.4 Since $\mathrm{P}^{2} \rightarrow \mathbb{R}^{3}$, there exists an axial map $q$ : $\mathrm{P}^{2} \times \mathrm{P}^{2} \rightarrow \mathrm{P}^{3}$. Note that $2<3<2 \cdot 2=4$. However, $q$ is not homotopic to a submersion. In fact, there does not exist any submersion $\mathrm{P}^{2} \times \mathrm{P}^{2} \rightarrow \mathrm{P}^{3}$, by the following easy argument involving Stiefel-Whitney classes: Suppose $g: \mathrm{P}^{2} \times \mathrm{P}^{2} \rightarrow \mathrm{P}^{3}$ is a submersion. Then we obtain the short exact sequence of vector bundles over $\mathrm{P}^{2} \times \mathrm{P}^{2}$

$$
0 \rightarrow E \rightarrow T\left(\mathrm{P}^{2} \times \mathrm{P}^{2}\right) \stackrel{d g}{\rightarrow} g^{*} T\left(\mathrm{P}^{3}\right) \rightarrow 0,
$$

where the kernel $E$ is a real line bundle. It then follows that

$$
w\left(\mathrm{P}^{2} \times \mathrm{P}^{2}\right)=w(E) g^{*} w\left(\mathrm{P}^{3}\right)=w(E)
$$

(the latter equality since $\mathrm{P}^{3}$ is parallelizable). But this is impossible since, for example, $w_{2}\left(\mathrm{P}^{2} \times \mathrm{P}^{2}\right) \neq 0$. 
Remark 3.5 It is possible to prove the upper bound in Theorem 3.3 by applying [11, Theorem 3] to an axial map $\mathrm{P}_{\bar{n}} \times \mathrm{P}_{\bar{n}} \rightarrow \mathrm{P}^{2|\bar{n}|-n_{1}+1}$ and noticing that the canonical inclusion $\mathrm{P}_{\bar{n}} \hookrightarrow \mathrm{P}^{2|\bar{n}|-n_{1}+1}$ is an $n_{1}$-equivalence. We have chosen the approach in [16] due to the intrinsic interest of Proposition 3.2.

Remark 3.6 The standard upper bound TC $\leq 2$ dim means that, in general, there are up to twice $\operatorname{dim}(X)$ classical homotopy obstructions to consider when bounding $\operatorname{TC}(X)$ from above. For instance, the top two are central in [6], with the very top one being critical for Costa-Farber's applications - the next-to-the-top one comes for free from Berstein [4]. Thus, the upper bound in Theorem 3.3 is already taking care of the first $n_{1}-1$ of these obstructions for $X=\mathrm{P}_{\bar{n}}$. But actually many more obstructions are dealt with by Theorem 3.8 below.

The problem of explicitly computing the exact value of the topological complexity of real projective spaces (ie the immersion problem of those manifolds) is an extremely difficult one, nowadays lacking of even a concrete guess on how the numerical solution should be. Indeed, not only can the gap between known upper and lower bounds be arbitrarily large, but the most accurate known information available today applies only to limited families of projective spaces (those whose dimension has a rather short dyadic expansion). It is in this sense that a general result about the computation of the topological complexity of projective product spaces cannot be expected to be more accurate than what happens for its special case of real projective spaces. Nonetheless, we next discuss a number of cases for which the general bounds in Theorem 3.3 can be largely improved. In particular, Proposition 3.7 below describes the precise value of the topological complexity of an infinite family of projective product spaces $\mathrm{P}_{\bar{n}}$ (those where $n_{1}$ is a 2 -power with $n_{1}$ dividing twice $n_{i}+1$ for $i>1$ ).

The lower bound in Theorem 3.3 ignores information which comes from the product $S^{n_{2}} \times \cdots \times S^{n_{r}}$. For instance, [10, Theorem 4.5], (5) and 'zero-divisors' cup-length (zcl) considerations (as defined by Farber in [8]) easily yield

$$
\operatorname{TC}\left(\mathrm{P}_{\bar{n}}\right) \geq 2^{e+1}+\ell(\bar{n})-2, \text { provided } n_{1} \geq 2^{e},
$$

which improves by an arbitrarily large amount the lower bound in Theorem 3.3 when $\ell(\bar{n}) \gg 0$. On the other hand, the general philosophy behind (1) implies that the lower bound in Theorem 3.3 can be much stronger than that in (13) if $\ell(\bar{n})=2$. For instance James [20] gives

$$
\operatorname{TC}\left(\mathrm{P}_{\left(2^{e}-1,2^{e}-1\right)}\right) \geq \mathrm{TC}\left(\mathrm{P}^{2^{e}-1}\right) \geq 2^{e+1}-2 e-(2,1,1,3),
$$

provided $e \equiv(0,1,2,3) \bmod 4$, a bound which is almost twice that in (13). Of course, further results of this sort can be deduced from our current knowledge of the 
immersion dimension of (usual) real projective spaces. In view of the first and third authors and Velasco-Fuentes [15, Theorems 2.1 and 2.4], it should be possible to use zcl-considerations based on generalized cohomology theories in order to insert the nice $\ell(\bar{n})$-feature of (13) into the lower bound in Theorem 3.3, thus merging the corresponding strengths of (13) and (14) into a single lower bound (we hope to explore such a possibility elsewhere).

More interesting is the fact that $\operatorname{TC}\left(\mathrm{P}_{\bar{n}}\right)$ can be arbitrarily smaller than the dimension of $\mathrm{P}_{\bar{n}}$. The simplest of such situations originates from the subadditivity of TC [8], as $\mathrm{TC}\left(\mathrm{P}_{\bar{n}}\right)-\mathrm{TC}\left(\mathrm{P}_{\bar{m}}\right) \leq 2$ whenever $\mathrm{P}_{\bar{n}} \approx \mathrm{P}_{\bar{m}} \times S^{n_{i}}$ (the latter decomposition is characterized arithmetically in [7, Theorem 2.20]). As an extreme situation consider the following partial analogue of [7, (2.21)].

Proposition 3.7 Let $\phi\left(n_{1}\right)$ be the number of positive integers less than or equal to $n_{1}$ which are congruent to $0,1,2$, or $4(\bmod 8)$. If $v\left(n_{i}+1\right) \geq \phi\left(n_{1}\right)$ for all $i>1$, then

$$
\operatorname{zcl}_{\mathbb{Z}_{2}}\left(\mathrm{P}^{n_{1}}\right)+\ell(\bar{n})-1 \leq \mathrm{TC}\left(\mathrm{P}_{\bar{n}}\right) \leq \mathrm{TC}\left(\mathrm{P}^{n_{1}}\right)+\ell(\bar{n})-1 .
$$

Further, both inequalities above become equalities precisely for $n_{1}$ a 2 -power.

Proof The first inequality is (13); the second inequality follows from [7, Theorem 2.20]. The final assertion follows from the standard fact that $\mathrm{TC}\left(\mathrm{P}^{n_{1}}\right)=\mathrm{zcl}_{\mathbb{Z}_{2}}\left(\mathrm{P}^{n_{1}}\right)$ precisely for $n_{1}$ a 2 -power.

Proposition 3.7 suggests the possibility that TC $\left(\mathrm{P}_{\bar{n}}\right)$ can be estimated for any $\bar{n}$ in terms of $\mathrm{TC}\left(\mathrm{P}^{n_{1}}\right)$ and $\ell(\bar{n})$ alone. Theorem 3.8 below (whose proof is postponed to the next section) fits into such a general philosophy, and shows that the low TC-phenomenon in Proposition 3.7 holds even if there are no spheres factoring out $\mathrm{P}_{\bar{n}}$.

Theorem 3.8 If $k$ denotes the number of spheres $S^{n_{i}}$ with $n_{i}$ even and $i>1$, then $\mathrm{TC}\left(\mathrm{P}_{\bar{n}}\right)<\left(\mathrm{TC}\left(\mathrm{P}^{n_{1}}\right)+1\right)(\ell(\bar{n})+k)$.

The upper bound in Theorem 3.8 will be much lower than the dimension of $\mathrm{P}_{\bar{n}}$ provided the sum $n_{2}+\cdots+n_{r}$ is large enough-which can hold even if there are no spheres $S^{n_{i}}$ factoring out $\mathrm{P}_{\bar{n}}$. Thus, in such cases, most of the homotopy obstructions in the motion planning problem for $\mathrm{P}_{\bar{n}}$ already vanish. It is worth noticing that $\mathrm{TC}\left(\mathrm{P}_{\bar{n}}\right)$ is not always less than $\operatorname{dim}\left(\mathrm{P}_{\bar{n}}\right)$ : If $1^{r}$ stands for the $r$-tuple $(1, \ldots, 1)$, then $\operatorname{TC}\left(\mathrm{P}_{1^{r}}\right)=\operatorname{dim}\left(\mathrm{P}_{1^{r}}\right)$, in view of Proposition 3.7. On the other hand, the upper bound in Theorem 3.8 does not always improve that in Theorem 3.3. For instance, in the case of $\mathrm{P}_{\left(2^{e}, 2^{e}\right)}$, the former bound is $6 \cdot 2^{e}$ while the latter one is only $3 \cdot 2^{e}+1$. 


\section{Equivariant topological complexity}

In a recent paper [5] Hellen Colman and the second author explore an equivariant generalization of topological complexity, in the setting of compact group actions. Here we give additional examples and facts which will be useful in applying their results to the estimation of topological complexity of projective product spaces.

Let $G$ be a compact Hausdorff topological group (in our present applications $G$ will be the cyclic group $\mathbb{Z}_{2}$ ). If $p: E \rightarrow B$ is a $G$-map, the equivariant sectional category of $p$, denoted $\operatorname{secat}_{G}(p)$, is defined in [5, Section 5] to be the least integer $k$ such that $B$ may be covered by $k G$-invariant open sets $U_{1}, \ldots, U_{k}$ on each of which there exists a $G$-homotopy section, that is a $G$-map $s_{i}: U_{i} \rightarrow E$ such that $p \circ s_{i}$ is $G$-homotopic to the inclusion $\iota_{U_{i}}: U_{i} \hookrightarrow B$. If $p$ is a $G$-fibration, then this is equivalent to requiring the existence of a $G$-section $s_{i}: U_{i} \rightarrow E$, ie a $G$-map $s_{i}$ such that $p \circ s_{i}=\iota_{U_{i}}$.

In particular, for any $G$-space $X$ the equivariant topological complexity of $X$ is defined in [5, Section 6] to be the equivariant sectional category of the double evaluation map $X^{[0,1]} \rightarrow X \times X$. Here $G$ acts diagonally on the product and by composition on the path space of $X$.

In keeping with the conventions in place in this paper, we will define the equivariant topological complexity to be one less than the number of sets in the open cover; thus

$$
\operatorname{TC}_{G}(X)=\operatorname{secat}_{G}\left(X^{[0,1]} \rightarrow X \times X\right)-1 .
$$

Lemma 4.1 Let $G=\mathbb{Z}_{2}$ act antipodally on the sphere $S^{n}$, where $n \geq 1$. Then

$$
\operatorname{TC}_{G}\left(S^{n}\right)= \begin{cases}1 & \text { if } n \text { is odd } \\ 2 & \text { if } n \text { is even }\end{cases}
$$

Proof We argue that the usual motion planning rules on the spheres can be made equivariant with respect to the antipodal action, by choosing vector fields which are equivariant.

Suppose $n$ is odd. Then the projective space $\mathrm{P}^{n}$ has zero Euler characteristic and so admits a nowhere-vanishing vector field. Using the double cover immersion $S^{n} \rightarrow P^{n}$, this pulls back to a nowhere-vanishing vector field $v$ on $S^{n}$ which is equivariant in the sense that $d g(v(A))=v(g A)$ for $g \in G$ and $A \in S^{n}$. We consider the open sets $U_{0}=\left\{(A, B) \in S^{n} \times S^{n} \mid A \neq-B\right\}$ and $U_{1}=\left\{(A, B) \in S^{n} \times S^{n} \mid A \neq B\right\}$. We define $s_{0}$ on $U_{0}$ by choosing the shortest geodesic path from $A$ to $B$ (traveled at constant velocity). We define $s_{1}$ on $U_{1}$ in two stages: First travel from $A$ to $-A$ along 
the great circle in the direction determined by $v(A)$; second travel from $-A$ to $B$ along the shortest geodesic path. It is easy to check that these sets and motion planning rules are $G$-invariant.

When $n$ is even, removing a point $[C]$ from $P^{n}$ gives an open manifold homotopy equivalent to $P^{n-1}$, which therefore admits a nowhere-vanishing vector field. Again we pull this back to obtain a nowhere-vanishing equivariant vector field $v^{\prime}$ on $S^{n}-\{-C, C\}$. We let $U_{0}$ and $s_{0}$ be as before. We let $U_{1}^{\prime}=\left\{(A, B) \in S^{n} \times S^{n} \mid A \neq B, C,-C\right\}$ and define $s_{1}^{\prime}$ using $v^{\prime}$ similarly to $s_{1}$. Finally we let $U_{2}^{\prime}=\{(A,-A) \mid A \in W \cup-W\}$, where $W$ is a small open disk neighbourhood centred on $C$. The path $s_{2}^{\prime}(A,-A)$ for $A \in W$ travels first along the geodesic segment to the centre $C$ of $W$; then along some fixed path $\gamma$ from $C$ to $-C$; then along the geodesic segment in $-W$ to $-A$. For $A \in-W$ the path $s_{2}^{\prime}(A,-A)$ travels first along the geodesic segment in $-W$ to $-C$; then along $-\gamma$ from $-C$ to $C$; then along the geodesic segment in $W$ to $-A$.

The lower bounds are given by the obvious inequality $\mathrm{TC}(X) \leq \mathrm{TC}_{G}(X)$, which holds for any $G$-space $X$.

Theorem 4.2 Let $G$ be a compact Lie group, and let $X$ and $Y$ be smooth $G-$ manifolds. Then

$$
\mathrm{TC}_{G}(X \times Y) \leq \mathrm{TC}_{G}(X)+\mathrm{TC}_{G}(Y),
$$

where $X \times Y$ is given the diagonal $G$-action.

Proof Let $\mathrm{TC}_{G}(X)=n$ and $\operatorname{TC}_{G}(Y)=m$. Suppose that $X \times X=U_{0} \cup \cdots \cup U_{n}$, where the $U_{i}$ are open invariant sets with $G$-sections $s_{i}: U_{i} \rightarrow X^{[0,1]}$. Suppose further that $Y \times Y=V_{0} \cup \cdots \cup V_{m}$, where the $V_{j}$ are open invariant sets with $G-$ sections $\sigma_{j}: V_{j} \rightarrow Y^{[0,1]}$. We can find a $G$-invariant partition of unity $\left\{f_{i}\right\}$ on $X \times X$ subordinate to $\left\{U_{i}\right\}$ (see Guillemin, Ginzburg and Karshon [17, Corollary B.33]). Likewise let $\left\{g_{j}\right\}$ be a $G$-invariant partition of unity on $Y \times Y$ subordinate to $\left\{V_{j}\right\}$.

The rest of the proof proceeds by direct analogy with the proof in the nonequivariant case given in [8, Theorem 11], hence is omitted.

Remark 4.3 Theorem 4.2 is certainly not the most general setting in which the product inequality holds. For instance, we believe it holds whenever $X$ and $Y$ are $G$-ENRs.

Corollary 4.4 Consider the diagonal action of $\mathbb{Z}_{2}$ on $S_{\bar{n}}=S^{n_{1}} \times \cdots \times S^{n_{r}}$. If $k$ denotes the number of spheres with $n_{i}$ even, then

$$
\mathrm{TC}_{G}\left(S_{\bar{n}}\right)=\ell(\bar{n})+k .
$$


Proof This follows from Lemma 4.1, Theorem 4.2, and [8, Theorem 13] as the first and third terms in

$$
\operatorname{TC}\left(S_{\bar{n}}\right) \leq \operatorname{TC}_{G}\left(S_{\bar{n}}\right) \leq \sum_{i=1}^{r} \operatorname{TC}_{G}\left(S^{n_{i}}\right)
$$

agree.

The main result we will apply from [5] gives an upper bound for the (nonequivariant) topological complexity of a Borel fibration in terms of the topological complexity of the base and the equivariant topological complexity of the fiber.

Theorem 4.5 ([5, Theorem 6.21]) Let $X$ be a $G$-space, and let $E \rightarrow B=E / G$ be a numerable principal $G$-bundle. Then

$$
\operatorname{TC}\left(X_{G}\right)<\left(\operatorname{TC}_{G}(X)+1\right)(\operatorname{TC}(B)+1),
$$

where $X_{G}=E \times_{G} X$ is the corresponding Borel space of $X$.

Proof of Theorem 3.8 Let $\bar{m}=\left(n_{2}, \ldots, n_{r}\right)$. Note $\mathrm{P}_{\bar{n}}$ can be thought of as the Borel space $S^{n_{1}} \times_{\mathbb{Z}_{2}} S_{\bar{m}}$. The result then follows from Corollary 4.4 and Theorem 4.5.

The argument in the proof of Theorem 3.8 can be used to give low upper bounds for the LS-category of projective product spaces (extending the phenomenon in [7, (2.21)] when $\mathrm{P}_{\bar{n}}$ has a full set of factoring spheres). Namely

$$
\operatorname{cat}\left(\mathrm{P}_{\bar{n}}\right)<\left(n_{1}+1\right) \ell(\bar{n}) .
$$

Since $\mathrm{TC} \leq 2 \mathrm{cat}$, we get in particular

$$
\mathrm{TC}\left(\mathrm{P}_{\bar{n}}\right)<2\left(n_{1}+1\right) \ell(\bar{n})-1,
$$

which improves on Theorem 3.8 only when $\mathrm{P}_{\bar{n}}$ comes from a product having 'enough' even dimensional spheres, ie $k \geq C_{n_{1}} \ell(\bar{n})$ where

$$
C_{n_{1}}=\frac{2 n_{1}+1-\mathrm{TC}\left(\mathrm{P}^{n_{1}}\right)}{\mathrm{TC}\left(\mathrm{P}^{n_{1}}\right)+1} .
$$

Note that, although $k \leq \ell(\bar{n}), C_{n_{1}} \ll 1$ for any 'generic' $n_{1}$.

Just as (16) and Theorem 3.8 may fail to improve the upper bound in Theorem 3.3, the bound in (15) is not always useful (for instance if all the $n_{i}$ are equal). For such cases it is worth keeping in mind that, in view of [4, Theorem 3.5], the standard estimate cat $\leq \operatorname{dim}$ is improved by the inequality $\operatorname{cat}\left(\mathrm{P}_{\bar{n}}\right)<\operatorname{dim}\left(\mathrm{P}_{\bar{n}}\right)$ provided $n_{1}>1$ and $\ell(\bar{n})>1$. 


\section{Some explicit motion planning algorithms}

Recall that a motion planning algorithm (MPA) on a space $X$ is a (perhaps noncontinuous) section $s: X \times X \rightarrow X^{[0,1]}$ for the double evaluation map $X^{[0,1]} \rightarrow X \times X$. There are several (equivalent, if $X$ is an ENR) ways $s$ can be characterized to be tame (see Farber [9, Definition 4.4 and Proposition 4.12]). For the purposes of this paper we require that there is a nested sequence of open sets

$$
\varnothing=U_{0} \subseteq U_{1} \subseteq U_{2} \subseteq \cdots \subseteq U_{k+1}=X \times X
$$

so that $s$ is continuous on each difference $U_{i+1}-U_{i}(0 \leq i \leq k)$. Thus, the minimal $k$ in any such decomposition of $X \times X$ is the topological complexity of $s$, whereas $\mathrm{TC}(X)$ is the minimal topological complexity of MPA's on $X$. Note that the above condition of tameness is obviously met when $s$ is continuous on each member of a covering $\left\{U_{i}\right\}_{1 \leq i \leq k+1}$ of $X \times X$.

In this section, we describe two ways of constructing tame MPA's of practical use on projective product spaces that realize some of the upper bounds in Section 3. We first use [9, Theorem 4.49] in order to spell out the argument used in the proof of Proposition 3.7. The algorithm that we will describe applies to those $\mathrm{P}_{\bar{n}}$ satisfying the conditions in that proposition. For concreteness we will focus on the case of $\mathrm{P}_{(2,3)}$, although the steps described by our algorithm can be applied regardless of whether $n_{1}$ is a 2-power (for instance, we may construct of an MPA on $\mathrm{P}_{(5,7)}$ of topological complexity eight, which is at most one unit away from being optimal in view of Proposition 3.7). Start with the easy MPA $s: S^{3} \times S^{3} \rightarrow\left(S^{3}\right)^{[0,1]}$ given so that $s(x, y)$ is the path at constant velocity following the geodesic from $x$ to $y$. Here we have to choose the geodesic in the direction of a fixed nowhere-zero vector field on $S^{3}$, if $x=-y$. It is standard that $s$ is optimal as its topological complexity is that of $S^{3}$. On the other hand, an equally optimal MPA $\sigma: \mathrm{P}^{2} \times \mathrm{P}^{2} \rightarrow\left(\mathrm{P}^{2}\right)^{[0,1]}$ is described in [10, proof of Proposition 6.3]. It is given in such a way that, for a pair of lines $\left(L_{1}, L_{2}\right) \in \mathrm{P}^{2} \times \mathrm{P}^{2}$, $\sigma\left(L_{1}, L_{2}\right)$ is the constant-speed rotation about the origin from $L_{1}$ to $L_{2}$ in the plane they generate so that

(a) the rotation swipes the acute angle, if $L_{1}$ and $L_{2}$ are not perpendicular (in particular, the rotation is in fact static for $L_{1}=L_{2}$ );

(b) the rotation projects to the canonical orientation on the $\left(x_{1}, x_{2}\right)$-plane, if $L_{1}$ and $L_{2}$ have different and nondegenerate projections onto that plane;

(c) the rotation projects to the canonical orientation on the $\left(x_{1}, x_{3}\right)$-plane, if $L_{1}$ and $L_{2}$ have different and nondegenerate projections onto that plane;

(d) $L_{1}$ and $L_{2}$ must have different and nondegenerate projections onto the $\left(x_{2}, x_{3}\right)$ plane, and the rotation projects to the canonical orientation on that plane. 
The proof of [9, Theorem 4.49] asserts that the cartesian product $(\sigma, s)$ gives an MPA on $\mathrm{P}^{2} \times S^{3}$. The required MPA $\zeta: \mathrm{P}_{(2,3)} \times \mathrm{P}_{(2,3)} \rightarrow\left(\mathrm{P}_{(2,3)}\right)^{[0.1]}$ then follows in terms of the homeomorphism

$$
\begin{aligned}
\mathrm{P}_{(2,3)} & \cong \mathrm{P}^{2} \times S^{3}, \\
{[x, y] } & \leftrightarrow([x], x y),
\end{aligned}
$$

noted in [7, part (1) of Theorem 2.20] where the product $x y$ refers to multiplication in the quaternions $\mathbb{H}$ (with $\mathbb{R}^{3} \hookrightarrow \mathbb{R}^{4}=\mathbb{H}$ being the inclusion onto the first three coordinates). Explicitly, given a 'time' parameter $t \in[0,1]$ and a pair $\left(\left[x_{1}, y_{1}\right],\left[x_{2}, y_{2}\right]\right) \in \mathrm{P}_{(2,3)} \times \mathrm{P}_{(2,3)}$,

$$
\zeta\left(\left[x_{1}, y_{1}\right],\left[x_{2}, y_{2}\right]\right)(t)=\left[a_{t}, \bar{a}_{t} b_{t}\right]
$$

where $a_{t} \in S^{2}$ is any representative for $\sigma\left(\left[x_{1}\right],\left[x_{2}\right]\right)(t) \in \mathrm{P}^{2}, \bar{a}_{t}$ is the quaternion conjugate of $a_{t}$, and $b_{t}=s\left(x_{1} y_{1}, x_{2} y_{2}\right)(t) \in S^{3}$. The optimality of $\zeta$ is guaranteed by Proposition 3.7. The corresponding nested open sets in (17) can be explicitly described in terms of (18) and (a)-(d) using [9, Theorem 4.49]; the verification of the easy details is left to the reader (cf [10, Section 9]).

Next we describe an MPA realizing the upper bound given by Theorem 3.8. To fix ideas, we focus on the case of $\mathrm{P}_{\left(n_{1}, n_{2}\right)}$ with $n_{2}$ odd, assuming an MPA for $\mathrm{P}^{n_{1}}$ is given via a nonsingular map

$$
\phi=\left(\phi_{1}, \ldots, \phi_{k+1}\right): \mathbb{R}^{n_{1}+1} \times \mathbb{R}^{n_{1}+1} \rightarrow \mathbb{R}^{k+1}
$$

through the explicit construction in [10]. Thus, $\phi_{1}$ is assumed to be positive on the diagonal (explicit examples of nonsingular maps can easily be obtained from Lam [21] and Milgram [26]; some of them with $\left.\mathrm{TC}\left(\mathrm{P}^{n_{1}}\right)=k\right)$, and we consider the open covering $\left\{V_{i}\right\}_{i=1, \ldots, k+1}$ of $\mathrm{P}^{n_{1}} \times \mathrm{P}^{n_{1}}$ where $V_{i}$ consists of the pairs $([u],[v])$ with $\phi_{i}(u, v) \neq 0$ and, if $i>1,[u] \neq[v]$. (For instance, the four local motion planners in items (a)-(d) above arise when (19) is the restriction to $\mathbb{R}^{3} \times \mathbb{R}^{3}$ of the quaternionic 'twisted' multiplication $x \bar{y})$. Likewise $\mathrm{P}_{\left(n_{1}, n_{2}\right)} \times \mathrm{P}_{\left(n_{1}, n_{2}\right)}$ is covered by the sets $U_{i}$ given as the inverse image of the corresponding $V_{i}$ under the fibration $p \times p: \mathrm{P}_{\left(n_{1}, n_{2}\right)} \times \mathrm{P}_{\left(n_{1}, n_{2}\right)} \rightarrow \mathrm{P}^{n_{1}} \times \mathrm{P}^{n_{1}}$, where $p$ is as in (4).

In these conditions, the first main ingredient in the construction of the MPA we want is given by the maps $F_{i}: U_{i} \times[0,1] \rightarrow \mathrm{P}_{\left(n_{1}, n_{2}\right)} \times \mathrm{P}_{\left(n_{1}, n_{2}\right)}$ defined by

$$
F_{i}([u, r],[v, s], t)=\left(\left[u_{t}^{\prime \prime}, r^{\prime}\right],[v, s]\right),
$$


where we choose representatives $\left(u^{\prime}, r^{\prime}\right)$ and $\left(v^{\prime}, s^{\prime}\right)$ such that $\phi_{i}\left(u^{\prime}, v^{\prime}\right)>0$ (this choice is not canonical; we may equally well choose $\left(-u^{\prime},-r^{\prime}\right)$ and $\left.\left(-v^{\prime},-s^{\prime}\right)\right)$ and, in these terms, $u_{t}^{\prime \prime}$ is the unit vector obtained at time $t$ from the constant-speed rotation from $u^{\prime}$ to $v^{\prime}$ in the plane they span. Each $F_{i}$ is a homotopy, starting at the inclusion $U_{i} \hookrightarrow \mathrm{P}_{\left(n_{1}, n_{2}\right)} \times \mathrm{P}_{\left(n_{1}, n_{2}\right)}$, and finishing at a map $f_{i}: U_{i} \rightarrow \mathrm{P}_{\left(n_{1}, n_{2}\right)} \times \mathrm{P}_{\left(n_{1}, n_{2}\right)}$ whose image is contained in $(p \times p)^{-1}\left(\Delta_{\mathrm{P}_{1} n_{1}}\right)$, the inverse image under $p \times p$ of the diagonal $\mathrm{P}^{n_{1}} \hookrightarrow \mathrm{P}^{n_{1}} \times \mathrm{P}^{n_{1}}$. The second (and final) main ingredient in the construction of the MPA we want arises from the open covering $\left\{W_{0}, W_{1}\right\}$ of $(p \times p)^{-1}\left(\Delta_{\mathrm{P}^{n_{1}}}\right)$ where $W_{0}$ consists of pairs of the form $([v, s],[v, t])$ with $s \neq t$, and $W_{1}$ consists of pairs of the form $([v, s],[v, t])$ with $s \neq-t$. Then, our explicit MPA on $\mathrm{P}_{\left(n_{1}, n_{2}\right)}$ is defined at $([u, r],[v, s]) \in f_{i}^{-1}\left(W_{j}\right)$ by first moving $[u, r]$ to $\left[v, r^{\prime}\right]$, the first coordinate of $F_{i}([u, r],[v, s], 1)$, via the path $t \mapsto F_{i}([u, r],[v, s], t)$, and then moving $\left[v, r^{\prime}\right]$ to $[v, s]$ by means of the MPA from $r^{\prime}$ to $s$ explicitly described in the first half of the proof of Lemma 4.1. The last part of the motion is well defined due to its equivariance at the level of representatives.

\section{Appendix A Nonsingular maps on projective product spaces}

Just as for regular real projective spaces, the existence of axial maps for projective product spaces can be translated into the existence of certain nonsingular maps. Such a topic has been left out from the main body of the paper since, on the one hand, the construction is a straightforward generalization of the corresponding well-known property for usual projective spaces and, on the other, the language of nonsingular maps turns out to be irrelevant for the purposes of the paper since, as discussed in Section 2, they fail to provide sharp local motion planners as in the classical case [10]. Consequently, the ideas are loosely treated in this appendix, which has been included only for the sake of completeness.

There are two closely related notions of nonsingular maps associated to an axial map between projective product spaces. In the first one, for an $\ell$-tuple $\bar{q}=\left(q_{1}, \ldots, q_{\ell}\right)$, we consider the cone $Q_{\bar{q}}$ in $\mathbb{R}^{q_{1}+1} \times \cdots \times \mathbb{R}^{q_{\ell}+1}$ consisting of tuples $\bar{x}=\left(x_{1}, \ldots, x_{\ell}\right)$ with $\left|x_{1}\right|=\cdots=\left|x_{\ell}\right|$. Thus, $\mathrm{P}_{\bar{q}}$ is the projectivization of $Q_{\bar{q}}$, ie $\mathrm{P}_{\bar{q}}$ is the subspace of $\mathrm{P}^{|\bar{q}|+\ell-1}$ consisting of the lines contained in $Q_{\bar{q}}$. Then, a continuous map $f: Q_{\bar{n}} \times Q_{\bar{m}} \rightarrow \mathbb{R}^{k+1}$ is said to be nonsingular if $f(\lambda \bar{x}, \mu \bar{y})=\lambda \mu f(\bar{x}, \bar{y})$ for $\lambda, \mu \in \mathbb{R}$, and if the equality $f(\bar{x}, \bar{y})=0$ holds only with $\bar{x}=0$ or $\bar{y}=0$. With this definition, there is a one-to-one correspondence between the set of nonsingular maps $f: Q_{\bar{n}} \times Q_{\bar{m}} \rightarrow \mathbb{R}^{k+1}$ (taken up to multiplication by a nonzero scalar) and the set of axial maps $g: \mathrm{P}_{\bar{n}} \times \mathrm{P}_{\bar{m}} \rightarrow \mathrm{P}^{k}$. Such a corresponding pair $(f, g)$ fits in a 
commutative diagram

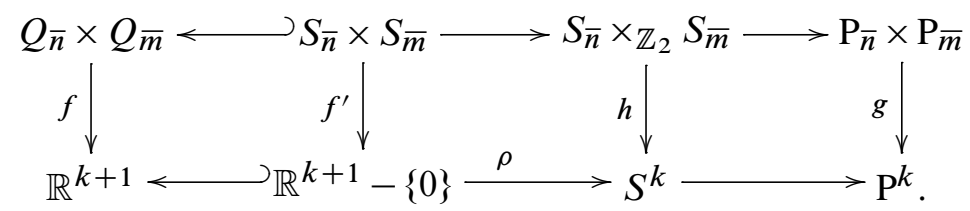

Here the unlabelled horizontal maps facing east are the obvious two-fold coverings, $\rho$ is the normalization map $\rho(u)=u /|u|, f^{\prime}$ is the restriction $\left.f\right|_{S_{\bar{n}} \times S_{\bar{m}}}$, and the right hand square is a pullback (hence $h$ is $\mathbb{Z}_{2}$-equivariant). Explicitly, given $f, g([\bar{x}],[\bar{y}])$ is the line in $\mathbb{R}^{k+1}$ that goes through the origin and $f(\bar{x}, \bar{y})$. Conversely, given $g$, pick $h$ as in the diagram above and precompose it with the double covering $S_{\bar{n}} \times S_{\bar{m}} \rightarrow S_{\bar{n}} \times \mathbb{Z}_{2} S_{\bar{m}}$ to get a $\mathbb{Z}_{2}$-biequivariant map $\tilde{g}: S_{\bar{n}} \times S_{\bar{m}} \rightarrow S^{k}$. Then $f$ is the 'biradial' extension of $\tilde{g}$ given by

$$
f(\bar{x}, \bar{y})= \begin{cases}\frac{|\bar{x}|}{\sqrt{\ell(\bar{n})}} \frac{|\bar{y}|}{\sqrt{\ell(\bar{m})}} \widetilde{g}\left(\frac{\sqrt{\ell(\bar{n})}}{|\bar{x}|}, \frac{\sqrt{\ell(\bar{m})}}{|\bar{y}|}\right), & \text { if } \bar{x} \neq 0 \text { and } \bar{y} \neq 0, \\ 0, & \text { if } \bar{x}=0 \text { or } \bar{y}=0 .\end{cases}
$$

Note that if $f: \mathbb{R}^{n_{1}+1} \times \mathbb{R}^{m_{1}+1} \rightarrow \mathbb{R}^{k+1}$ is a nonsingular map (in the usual sense), then for any $\bar{n}=\left(n_{1}, n_{2}, \ldots, n_{r}\right)$ and $\bar{m}=\left(m_{1}, m_{2}, \ldots, m_{s}\right)$ a nonsingular map $Q_{\bar{n}} \times Q_{\bar{m}} \rightarrow \mathbb{R}^{k+1}$ can be defined by $(\bar{x}, \bar{y}) \mapsto f\left(x_{1}, y_{1}\right)$. Of course, this fact is compatible with Remark 2.2.

A slight variation of the notion of nonsingular maps goes as follows: First we set $V_{\bar{t}}=\mathbb{R}^{t_{1}+1} \times \cdots \times \mathbb{R}^{t_{\ell}+1}$. A map $f: V_{\bar{n}} \times V_{\bar{m}} \rightarrow \mathbb{R}^{k+1}$ is said to be nonsingular if $f(\lambda \bar{x}, \mu \bar{y})=\lambda \mu f(\bar{x}, \bar{y})$ for $\lambda, \mu \in \mathbb{R}$, and if the equality $f(\bar{x}, \bar{y})=0$ holds only when a coordinate $x_{i}$ of $\bar{x}$ or a coordinate $y_{j}$ of $\bar{y}$ vanishes. Then the above considerations apply basically without change, except that (21) takes the slightly more elaborate form

$$
f(\bar{x}, \bar{y})= \begin{cases}N(\bar{x}, \bar{y}) \widetilde{g}\left(\frac{x_{1}}{\left|x_{1}\right|}, \ldots, \frac{x_{r}}{\left|x_{r}\right|}, \frac{y_{1}}{\left|y_{1}\right|}, \ldots, \frac{y_{r}}{\left|y_{s}\right|}\right), & \text { if no } x_{i} \text { nor } y_{j} \text { is zero, } \\ 0, & \text { otherwise }\end{cases}
$$

where $N(\bar{x}, \bar{y})=\left(\left|x_{1}\right| \cdots\left|x_{r}\right|\right)^{\frac{1}{r}}\left(\left|y_{1}\right| \cdots\left|y_{s}\right|\right)^{\frac{1}{s}}$ and, as above, $r=\ell(\bar{n})$ and $s=\ell(\bar{m})$.

\section{References}

[1] J F Adams, Geometric dimension of bundles over $\mathrm{RP}^{n}$, from: "Proceedings of the International Conference on Prospects in Mathematics", Res. Inst. Math. Sci., Kyoto Univ., Kyoto (1974) 1-17 MR0482761

[2] J Adem, S Gitler, I M James, On axial maps of a certain type, Bol. Soc. Mat. Mexicana 17 (1972) 59-62 MR0336757 
[3] L Astey, D M Davis, J González, Generalized axial maps and Euclidean immersions of lens spaces, Bol. Soc. Mat. Mexicana 9 (2003) 151-163 MR1988595

[4] I Berstein, On the Lusternik-Schnirelmann category of Grassmannians, Math. Proc. Cambridge Philos. Soc. 79 (1976) 129-134 MR0400212

[5] H Colman, M Grant, Equivariant topological complexity, Algebr. Geom. Topol. 12 (2012) 2299-2316

[6] A Costa, M Farber, Motion planning in spaces with small fundamental groups, Commun. Contemp. Math. 12 (2010) 107-119 MR2649230

[7] D M Davis, Projective product spaces, J. Topol. 3 (2010) 265-279 MR2651360

[8] M Farber, Topological complexity of motion planning, Discrete Comput. Geom. 29 (2003) 211-221 MR1957228

[9] M Farber, Invitation to topological robotics, Zurich Lectures in Advanced Mathematics, European Mathematical Society (EMS), Zürich (2008) MR2455573

[10] M Farber, S Tabachnikov, S Yuzvinsky, Topological robotics: motion planning in projective spaces, Int. Math. Res. Not. 2003 (2003) 1853-1870 MR1988783

[11] J M García-Calcines, L Vandembroucq, Topological complexity and the homotopy cofibre of the diagonal map, to appear in Math. Z. (2012)

[12] C G Gibson, K Wirthmüller, A A du Plessis, E J N Looijenga, Topological stability of smooth mappings, Lecture Notes in Mathematics 552, Springer, Berlin (1976) MR0436203

[13] M Golubitsky, V Guillemin, Stable mappings and their singularities, Graduate Texts in Mathematics 14, Springer, New York (1973) MR0341518

[14] J González, Topological robotics in lens spaces, Math. Proc. Cambridge Philos. Soc. 139 (2005) 469-485 MR2177172

[15] J González, E Torres-Giese, M Velasco-Fuentes, in preparation

[16] M Grant, Topological complexity, fibrations and symmetry, Topology Appl. 159 (2012) 88-97 MR2852952

[17] V Guillemin, V Ginzburg, Y Karshon, Moment maps, cobordisms, and Hamiltonian group actions, Mathematical Surveys and Monographs 98, American Mathematical Society (2002) MR1929136

[18] A Haefliger, M W Hirsch, Immersions in the stable range, Ann. of Math. 75 (1962) 231-241 MR0143224

[19] M W Hirsch, Immersions of manifolds, Trans. Amer. Math. Soc. 93 (1959) 242-276 MR0119214

[20] I M James, On the immersion problem for real projective spaces, Bull. Amer. Math. Soc. 69 (1963) 231-238 MR0144355 
[21] K Y Lam, Construction of nonsingular bilinear maps, Topology 6 (1967) 423-426 MR0217805

[22] K Y Lam, Sectioning vector bundles over real projective spaces, Quart. J. Math. Oxford Ser. 23 (1972) 97-106 MR0296965

[23] K Y Lam, D Randall, Geometric dimension of bundles on real projective spaces, from: "Homotopy theory and its applications", (A Adem, R J Milgram, D C Ravenel, editors), Contemp. Math. 188, Amer. Math. Soc., Providence, RI (1995) 137-160 MR1349135

[24] G Lupton, J Scherer, Topological complexity of $H$-spaces, Proc. Amer. Math. Soc. 141 (2013) 1827-1838 MR3020869

[25] S Melikhov, Singular fibers of generic smooth maps of negative codimension Available at http://mathoverflow.net/questions/94404\#95597

[26] R J Milgram, Immersing projective spaces, Ann. of Math. 85 (1967) 473-482 MR0211412

[27] J Oprea, J Walsh, Quotient maps, group actions and Lusternik-Schnirelmann category, Topology Appl. 117 (2002) 285-305 MR1874091

[28] B J Sanderson, A non-immersion theorem for real projective space, Topology 2 (1963) 209-211 MR0151987

[29] A Verona, Stratified mappings-structure and triangulability, Lecture Notes in Mathematics 1102, Springer, Berlin (1984) MR771120

JG, MX: Departamento de Matemáticas, Centro de Investigación y de Estudios Avanzados del IPN

A.P. 14-740, México City 07000, México

School of Mathematical Sciences, The University of Nottingham

University Park, Nottingham NG7 2RD, UK

Departamento de Matemáticas, Universidad de Guanajuato

Guanajuato, Gto 36000, México

jesus@math.cinvestav.mx, mark.grant@nottingham.ac.uk,

enrique.torres@cimat.mx, xico@math.cinvestav.mx

http://chucha.math.cinvestav.mx/,

http://www.maths.nottingham.ac.uk/personal/pmzmg/

Received: 8 August 2012 Revised: 18 November 2012 